\title{
Absolute angle-differential vibrational excitation cross sections for electron collisions with diacetylene
}

\author{
M. Allan, O. May, J. Fedor, and B. C. Ibănescu \\ Department of Chemistry, University of Fribourg, Chemin du Musée 9, CH-1700 Fribourg, Switzerland \\ L. Andric \\ UPMC, Université Paris 06, LCPMR, 11 rue Pierre et Marie Curie, 75231 Paris Cedex 05, France and \\ CNRS, LCPMR (UMR 7614), 11 rue Pierre et Marie Curie, 75231 Paris Cedex 05, France
}

\begin{abstract}
Absolute vibrational excitation cross sections were measured for diacetylene (1,3-butadiyne). The selectivity of vibrational excitation reveals detailed information about the shape resonances. Excitation of the $\mathrm{C} \equiv \mathrm{C}$ stretch and of double quanta of the $\mathrm{C}-\mathrm{H}$ bend vibrations reveals a ${ }^{2} \Pi_{u}$ resonance at $1 \mathrm{eV}$ (autodetachment width $\sim 30 \mathrm{meV}$ ) and $\mathrm{a}^{2} \Pi_{g}$ resonance at $6.2 \mathrm{eV}$ (autodetachment width $1-2 \mathrm{eV}$ ). There is a strong preference for excitation of even quanta of the bending vibration. Excitation of the $\mathrm{C}-\mathrm{H}$ stretch vibration reveals $\sigma^{*}$ resonances at $4.3,6.8$, and $9.8 \mathrm{eV}$, with autodetachment widths of $\sim 2 \mathrm{eV}$. Detailed information about resonances permits conclusions about the mechanism of the dissociative electron attachment.
\end{abstract}

\section{INTRODUCTION}

Diacetylene (1,3-butadiyne, $\mathrm{H}-\mathrm{C} \equiv \mathrm{C}-\mathrm{C} \equiv \mathrm{C}-\mathrm{H})$, and electron collisions with it, occur in a number of practical environments. Diacetylene is of interest for astronomy-it is a potential precursor for the $\mathrm{H}-\mathrm{C} \equiv \mathrm{C}-\mathrm{C} \equiv \mathrm{C}^{-}$anion, recently detected in interstellar space [1,2] and it was detected in the upper layers of planetary atmospheres (Titan, Uranus) $[3,4]$, where free electrons also occur. Electron collisions with diacetylene are of interest also in technological plasmas $[5,6]$. Diacetylene is an important intermediate in flames [7,8], in particular, for the formation of soot [9]. These applications, with emphasis on electron-induced chemical change, motivated our earlier study of absolute dissociative electron attachment (DEA) cross sections [10]. Diacetylene is an unusual molecule in terms of being carbon and energy rich, and being a relatively long linear molecule. It was one of the first polyatomic molecules for which luminescence of the positive ion was detected [11].

The present study reports absolute elastic and vibrational excitation (VE) cross sections. Apart from the inherent interest in these cross sections, justified by the occurrence of diacetylene in various plasmas, the present results yield information on the resonant structure and are helpful for understanding DEA.

Resonant electron-induced processes start with a vertical attachment of an electron to the molecule-the anion initially has the geometry of the target. The anion then starts to relax, and it distorts as a consequence of modifications of bonding caused by the extra electron. The distortion may be minor, for example, the $\mathrm{C} \equiv \mathrm{C}$ bond lengthens because the $\pi^{*}$ orbital into which the extra electron is accommodated is antibonding along the $\mathrm{C} \equiv \mathrm{C}$ bond, or less trivial, such as the symmetry lowering (Renner-Teller effect) related to vibronic coupling [12], the focus of interest in the present study. In the process of this relaxation, autodetachment proceeds at a rate given by the width $\Gamma$ of the resonance at each particular geometry, leading generally to a vibrationally excited state of the neutral molecule. The selectivity into which vibrational mode the resonance decays, and how many quanta are excited, are indicative of what geometry change is occurring during the relaxation. (See Ref. [13] for a pioneering study.) DEA is competing with the VE; it can be regarded to be due to those anions which survived, did not autodetach, and whose geometry has distorted beyond the stabilization point- the crossing of the anion and neutral potential surfaces. The information about the path of the relaxation, derived from the VE, can thus help in understanding DEA.

An early study of VE in diacetylene [14] found that bending vibration is strongly excited. In addition, it reported a strong propensity for exciting double quanta of the bending vibration. The limitation of that study was that the magnetically collimated spectrometer used could not measure angular distributions-it measured the superposition of $0^{\circ}$ and $180^{\circ}$ cross sections. A question thus remained as to whether the propensity does not apply only to forward and backward scattering. The present instrument can measure the entire angular distribution, derive integral cross sections, and remove the ambiguity.

\section{EXPERIMENT}

The measurements were performed using a spectrometer with hemispherical analyzers [15-18]. The energy resolution was $\sim 15 \mathrm{meV}$ in the energy-loss mode, at a beam current of $\sim 200 \mathrm{pA}$. The energy of the incident beam was calibrated on the $19.365-\mathrm{eV}$ [19] ${ }^{2} S$ resonance in helium and is accurate to within $\pm 10 \mathrm{meV}$. The instrumental response function was determined on elastic scattering in helium and all spectra were corrected as described earlier [16,18]. Absolute values of the cross sections were determined by the relative flow technique as described by Nickel et al. [20] using the theoretical helium elastic cross sections of Nesbet [21] as a reference. The confidence limit is approximately $\pm 20 \%$ for the elastic cross sections and $\pm 25 \%$ for the inelastic cross sections (two standard deviations). The butadiyne and helium pressures in the gas inlet line were typically 0.08 and 0.24 mbars, respectively, during the absolute measurements. (Other spectra were measured with a pressure of $0.5-1.0$ mbars in the inlet line and $5 \times 10^{-7}$ mbars in the main chamber.) The background 
was determined by a recording signal with gas flowing into the main chamber via a bypass line and not the nozzle. This background was generally negligible except in the more forward scattering and at low energies-but for consistency the "by-pass signal" was subtracted even when it was very low.

The angular distributions were measured using a combined mechanical setting of the analyzer and magnetic deflection using the magnetic angle changer [22,23], correcting the curves for the instrumental response function, and fitting them to the discrete absolute values measured at $20^{\circ}, 45^{\circ}$, $90^{\circ}, 135^{\circ}$, and $180^{\circ}$, as described in Refs. [16] and [18]. The angle of the magnetic scan was incremented in steps of $2.5^{\circ}$. Quantum chemical calculations were performed with the Firefly quantum chemical package [24], which is partially based on the GAMESS (US) [25] source code.

\section{RESULTS AND DISCUSSION}

\section{A. Electron energy-loss spectra}

The first step in the characterization of resonances using vibrational excitation is the determination of which vibrational modes play a key role. This can be seen in the energy-loss spectra shown in Fig. 1. The strongest inelastic peak in the spectrum recorded with an incident electron energy situated within the lowest ${ }^{2} \Pi_{u}$ shape resonance [14], at $E_{i}=1 \mathrm{eV}$, is the excitation of $v_{2}$, the symmetric $\mathrm{C} \equiv \mathrm{C}$ stretch vibration. (see Table I for a list of the vibrations) This is consistent with the assignment of the resonance to a temporary occupation of the $\pi_{u}$ orbital, an in-phase combination of the antibonding $\mathrm{C} \equiv \mathrm{C}$ $\pi^{*}$ orbitals, strongly antibonding with respect to the $\mathrm{C} \equiv \mathrm{C}$ distance.

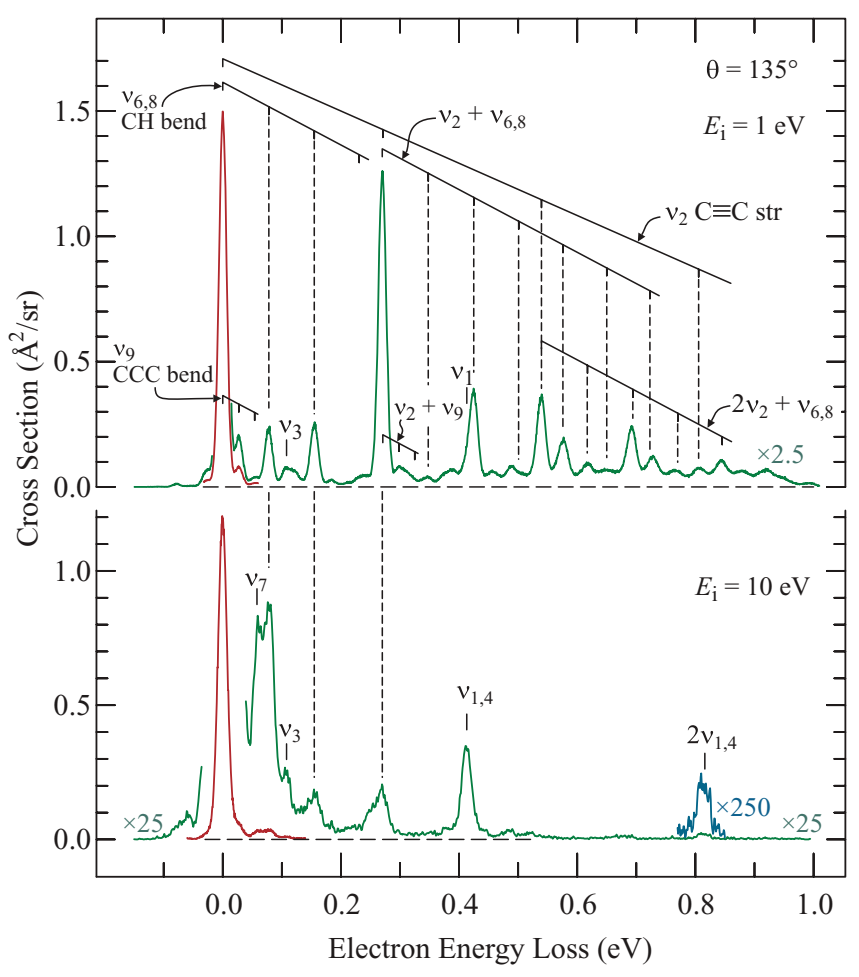

FIG. 1. (Color online) Electron energy-loss spectra recorded at constant incident energies of 1 and $10 \mathrm{eV}$ and a scattering angle of $135^{\circ}$.
TABLE I. Vibrational frequencies of diacetylene, with infrared activities indicated as inactive (ia), very weak (VW), medium (M), and very strong (VS) (from Refs. [27] and [28]).

\begin{tabular}{ccccc}
\hline \hline No. & Sym. & Type & Energy $(\mathrm{meV})$ & IR activity \\
\hline$\nu_{1}$ & $\sigma_{g}^{+}$ & C - H str & 413 & ia \\
$\nu_{2}$ & $\sigma_{g}^{+}$ & C $\equiv$ C str & 271 & ia \\
$\nu_{3}$ & $\sigma_{g}^{+}$ & C - C str & 108 & ia \\
$\nu_{4}$ & $\sigma_{u}^{+}$ & C - H str & 412 & VS \\
$\nu_{5}$ & $\sigma_{u}^{+}$ & C $\equiv$ C str & 250 & M \\
$\nu_{6}$ & $\pi_{g}$ & C - H bend & 78 & ia \\
$\nu_{7}$ & $\pi_{g}$ & CCC bend & 60 & ia \\
$\nu_{8}$ & $\pi_{u}$ & C - H bend & 78 & VS \\
$\nu_{9}$ & $\pi_{u}$ & CCC bend & 27 & VW \\
\hline \hline
\end{tabular}

The majority of the remaining peaks are due to excitation of the $\mathrm{C}-\mathrm{H}$ bending vibrations, indicating that the ground-state anion is bent. density functional theory (DFT) calculations, both those of Ref. [26] and our own, confirm this, with the cis and trans isomers having about the same energy. Unfortunately, the two vibrations $v_{6}\left(\pi_{g}\right)$ and $\nu_{8}\left(\pi_{u}\right)$ cannot be distinguished with the present resolution-only their sum $v_{6,8}$ can be measured. Already at this stage a preference for the excitation of even quanta of $v_{6,8}$ can be discerned in the upper spectrum in Fig. 1, as already noted in the earlier study [14]. The effect is particularly clearly visible for $v_{6,8}$ combined with $v_{2}$, which is nearly exclusively resonant, where direct excitation of IR-active transitions does not interfere. The single quantum of $v_{8}$ is excited quite strongly by dipole (nonresonant) mechanism because of its IR activity.

Other vibrations excited are the CCC bending vibration $v_{9}$ (both alone and in combination with $v_{2}$ ), the $\mathrm{C}-\mathrm{C}$ stretch $v_{3}$, and the $\mathrm{C}-\mathrm{H}$ stretch $v_{1}$. The two $\mathrm{C}-\mathrm{H}$ stretch vibrations $v_{1}$ $\left(\sigma_{g}\right)$ and $\nu_{4}\left(\sigma_{u}\right)$ cannot be resolved and only their sum can be recorded. They overlap with the $v_{2}+2 v_{6}$ peak, but can be distinguished as an asymmetric shape of the observed band.

A spectrum recorded at $E_{i}=10 \mathrm{eV}$ is shown in Fig. 1 as an example of a spectrum obtained at higher energies. The progressions are much shorter, there is much less excitation of overtones and combination vibrations. This indicates a much shorter autodetachment lifetime of the resonances at higher energy, which permits less relaxation before autodetachment. The $\mathrm{C}-\mathrm{H}$ stretch vibration $v_{1,4}$ is more prominent than at $1 \mathrm{eV}-\mathrm{a}$ general phenomenon among hydrocarbons [13].

Figure 2 makes a preview of the role of scattering angle on the vibrational excitation in the $1-\mathrm{eV}$ resonance region. Direct (dipole) excitation of IR-active vibrations (which affects, as IR absorption, primarily the fundamentals and not overtones and combination vibrations) is prominent in the forward direction, leading to a giant $\left(21 \AA^{2} / \mathrm{sr}\right)$ cross section for the $\nu_{8}$ vibration. The resonant process is manifested even at $0^{\circ}$, though by the excitation of overtone and combination vibrations. There is a very clear evidence for only even quanta of the $\mathrm{C}-\mathrm{H}$ bending vibrations being resonantly excited, particularly when superimposed on one or two quanta of the $\mathrm{C} \equiv \mathrm{C}$ stretch vibration, that is, when the direct process does not interfere. In particular, note that the $v_{2}+v_{6,8}$ vibration is missing. Odd quanta of the bending vibration are present at $90^{\circ}$, but even here 


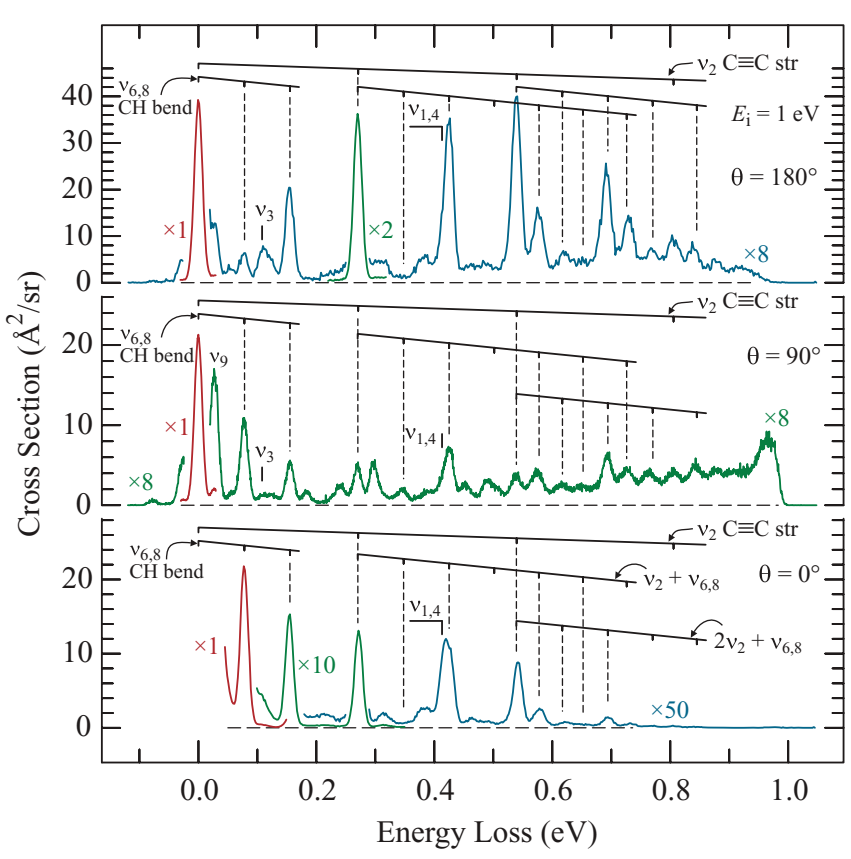

FIG. 2. (Color online) Electron energy-loss spectra recorded at a constant incident energy of $1 \mathrm{eV}$ and scattering angles of $0^{\circ}, 90^{\circ}$, and $180^{\circ}$.

they are weaker than even quanta. Otherwise, the spectrum is dominated by high bending overtones. Odd bending vibrations appear to be missing also in the $180^{\circ}$ spectrum; it is again particularly evident for the $v_{2}+v_{6,8}$ vibration, which has the advantage of not being obscured by any other peak. $v_{2}$ is generally more pronounced at $180^{\circ}$ than at $90^{\circ}$. High bending overtones become more prominent at higher-energy losses, particularly at $90^{\circ}$.

Figure 3 shows an energy-loss spectrum recorded with a slightly higher resolution $(12 \mathrm{meV})$ at a constant residual energy of $0.8 \mathrm{eV}$. Spectra recorded at constant residual energies are generally more suitable for observation of selectivity at higher-energy losses [29]. The preference for even quanta of the $\mathrm{C}-\mathrm{H}$ bend vibrations stands out clearly, even with simultaneous excitation of three quanta of $\mathrm{C} \equiv \mathrm{C}$ stretch.

The preference for the excitation of even quanta is related to the symmetry of the final vibrational state. (See also the discussion concerning OCS in Ref. [30]) According to the discussion of Schulz and Wong [31] and of Gallup [32,33], a given final vibrational state $\chi_{f}$ will be excited only when it has the same symmetry as the force field $G$ (the propagator in Ref. [31]). For the $\Pi_{u}$ resonance $G$ has the symmetry $\Pi_{u} \otimes \Pi_{u}=\Sigma_{g}\left(+\Delta_{g}\right)$, that is, apart from the $\Delta_{g}$ contribution, only totally symmetrical vibrational states will be strongly excited. Single quanta of C-H bending are $\pi_{u}\left(\nu_{6}\right)$ and $\pi_{g}\left(\nu_{8}\right)$ and will not be excited strongly. In contrast, even quanta of $\mathrm{C}-\mathrm{H}$ bending (both $v_{6}$ and $\nu_{8}$ ) result (among others) in a $\sigma_{g}$ vibrational state which has the correct symmetry. The situation is qualitatively illustrated in Fig. 4. The attachment promotes the initial wave packet onto the double-minimum potential surface of the resonance. It then relaxes by flowing into the two minima in a symmetrical manner. The relaxed wave function is projected onto the final vibrational states upon detachment. Only transitions into symmetrical final states are possible.

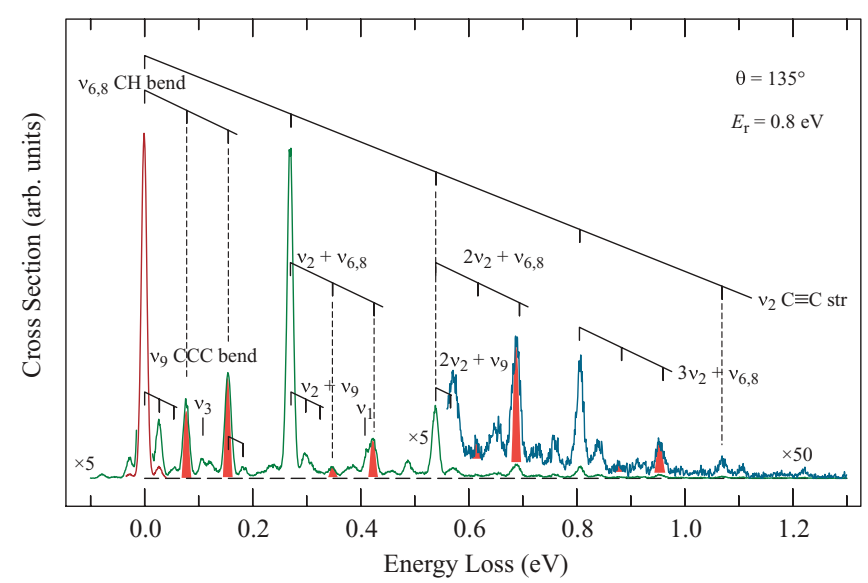

FIG. 3. (Color online) Electron energy-loss spectrum recorded at a slightly higher resolution (12 meV), at a constant residual energy of $0.8 \mathrm{eV}$ and a scattering angle of $135^{\circ}$. Odd and even quanta of $\mathrm{C}-\mathrm{H}$ bending are marked by (red) shading.

\section{B. Resonances}

Resonances, in particular, shape resonances, can be observed as enhancements of the vibrational excitation cross sections over relatively narrow energy ranges. A preview of the resonances is given in Fig. 5, where the role of the scattering angle is diminished (and the statistics is improved) by taking a sum of several angles. Indication of the nature of the resonances is given by the selectivity of the excitation [13]. The $\mathrm{C} \equiv \mathrm{C}$ stretch excitation cross section reveals resonances where the extra electron occupies an orbital antibonding with respect to the $\mathrm{C} \equiv \mathrm{C}$ bond, i.e., a $\pi^{*}$ orbital. Similarly, the $\mathrm{C}-\mathrm{H}$ stretch vibration selectively reveals $\sigma^{*}$ resonances, antibonding with respect to the $\mathrm{C}-\mathrm{H}$ bond. The $\pi^{*}$ orbitals do not have any coefficients on the $\mathrm{H}$ atoms and thus cannot be antibonding with respect to the $\mathrm{C}-\mathrm{H}$ bond.

The $\mathrm{C} \equiv \mathrm{C}$ stretch excitation in Fig. 5 reveals two resonances, at 1 and $6.2 \mathrm{eV}$, reported previously and assigned as ${ }^{2} \Pi_{u}$ and ${ }^{2} \Pi_{g}$ [14]. The $\mathrm{C}-\mathrm{H}$ stretch excitation reveals three new resonances at $4.3,6.8$, and $9.8 \mathrm{eV}$. The observed widths of the bands are a convolution of the autodetachment widths $\Gamma$, due to the fast autodetachment rate, and Franck-Condon widths, due to the distribution of the Franck-Condon factors, and related

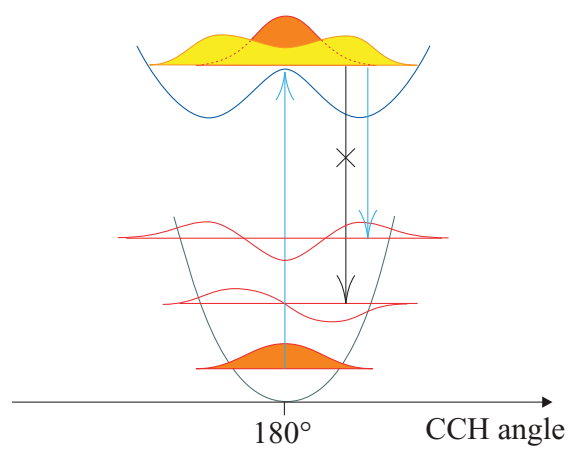

FIG. 4. (Color online) Schematic diagram of the relaxation of a vibrational wave packet on a double-minimum potential surface such as the lower branch of a ${ }^{2} \Pi_{u}$ resonance and the decay into one and two quanta of bending vibration. 


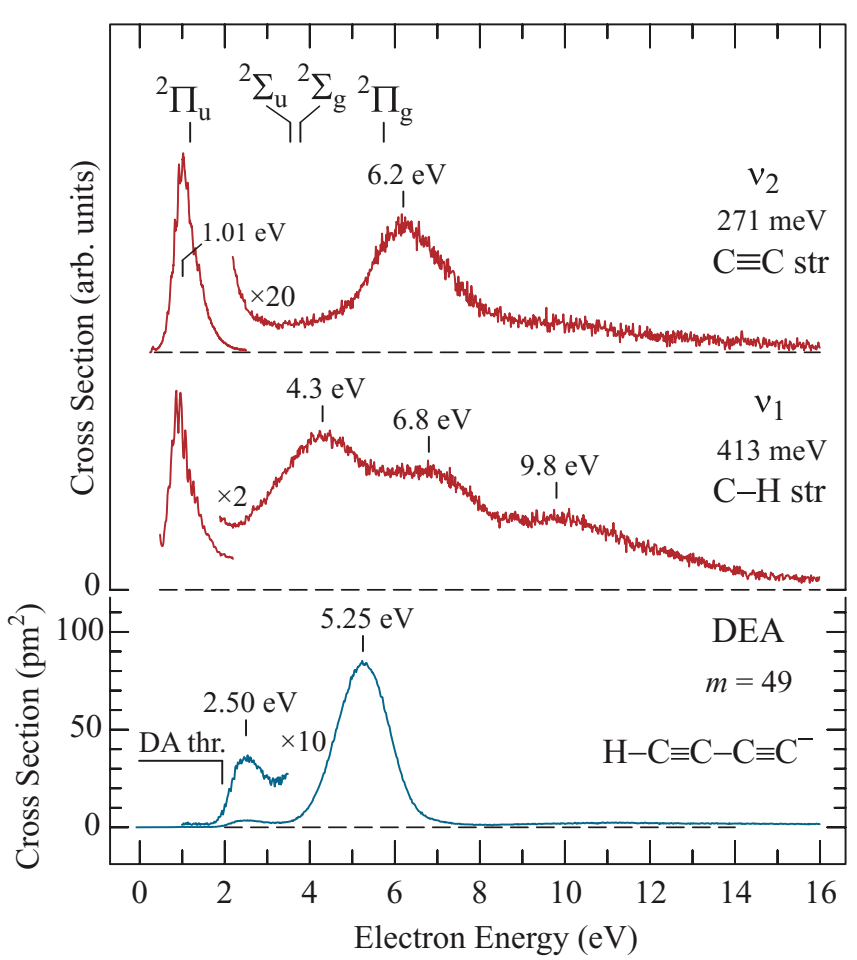

FIG. 5. (Color online) The top two curves show cross sections for exciting the $\mathrm{C}-\mathrm{H}$ and $\mathrm{C} \equiv \mathrm{C}$ stretch vibrations (sums of spectra recorded at $45^{\circ}, 135^{\circ}$, and $180^{\circ}$ ). Predictions of the scaled virtual orbital energies are shown on the top. The DEA spectrum (loss of $\mathrm{H}$ ) is shown at the bottom.

to the change of equilibrium geometry between the neutral target and the negative-ion resonance. The absence of any vibrational structure indicates that the observed widths, $\sim 2 \mathrm{eV}$, are in great part due to the autodetachment contribution for the 4.3-, 6.8-, and 9.8-eV bands. This is in contrast to the ${ }^{2} \Pi_{u}$ resonance at $1 \mathrm{eV}$, with a clear structure of vibrational origin (boomerang structure [34]), and with peaks as narrow as $30 \mathrm{meV}$ (see Sec.III D). The width of the ${ }^{2} \Pi_{u}$ band at $1 \mathrm{eV}$, $\sim 500 \mathrm{meV}$, is thus in its majority due to the Franck-Condon width and not to the autodetachment width. Assuming that the bands in the $\mathrm{C}-\mathrm{H}$ stretch excitation cross section have about the same Franck-Condon contribution to their total widths of $\sim 2 \mathrm{eV}$, leads to the conclusion that the total widths of the $\sigma^{*}$ resonances are dominated by their autodetachment widths, which are consequently $\sim 2 \mathrm{eV}$. (The $1-\mathrm{eV}^{2} \Pi_{u}$ resonance appears, relatively weakly, even in the $\mathrm{C}-\mathrm{H}$ stretch excitation curve in Fig. 5, but this could actually be due to the excitation of the $v_{2}+2 v_{6,8}$ vibration, which is partially overlapping with $v_{1}$-see Figs. 1 and 3.)

We use the method of empirically scaled virtual orbital energies (an extension of the Koopmans' theorem) as a guide in the assignment of the resonances [35,36]. This method is admittedly oversimplified, but has repeatedly proven to be a reliable guide for assigning shape resonances when a precision of $\sim 0.3-0.5 \mathrm{eV}$ is sufficient-as in the present case. An improved precision has been reported when scaling parameters were optimized for a certain class of compounds and for a certain type of orbitals $\left(\sigma^{*}, \pi^{*}\right)$ [37], but we prefer to use the scaling parameters of Chen and Gallup [36] for better consistency with our previous work. We point out that this scaling has correctly reproduced the shape resonances in a wide variety of compounds and even unusual types of orbitals, for example, both $\pi^{*}$ and $\mathrm{C}-\mathrm{Cl} \sigma^{*}$ resonances in chlorobenzene [38], a high-lying $(5.5 \mathrm{eV}) \sigma^{*}$ resonance in cyclopropane [39], and a very exotic $\sigma^{*}$ resonance in propellane [40]. This scaling is applicable to the SCF 6-31G* model (with geometry optimized within the same model) and its predictions for the lowest four virtual orbitals are indicated by bars at the top of Fig. 5. The validity of the model is confirmed by the excellent prediction for the ${ }^{2} \Pi_{u}$ resonance and the very satisfactory agreement for the ${ }^{2} \Pi_{g}$ resonance.

Two predicted shape resonances, with temporary occupation of the $\sigma_{u}$ and $\sigma_{g}$ orbitals, fit the first observed $\sigma^{*}$ band at $4.3 \mathrm{eV}$. Orbital diagrams shown in Fig. 6 indicate a strong antibonding character of both with respect to the $\mathrm{C}-\mathrm{H}$ bond, as indicated by the experiment. We cannot decide whether the resonance is ${ }^{2} \Sigma_{u}$ or ${ }^{2} \Sigma_{g}$. The only criterion is that the $\sigma_{g}$ orbital couples to the $s$ wave, providing a path for the electron to "leak out" without a barrier, and thus may not give rise to a resonance, whereas the $\sigma_{u}$ orbital, where the lowest wave is $p$, with a centrifugal barrier, can support a resonance. This argument gives a preference for the ${ }^{2} \Sigma_{u}$ assignment of the 4.3-eV band. We feel that the scaled virtual orbital energy method becomes unreliable at higher energies and do not attempt an assignment of the 6.8- and 9.8-eV $\sigma^{*}$ resonances.

\section{Relation to dissociative electron attachment}

Figure 5 shows that the $1-\mathrm{eV}^{2} \Pi_{u}$ resonance, at its center, cannot lead to DEA because it lies below the DEA threshold (at $1.94 \mathrm{eV}$ ). Direct dissociation by the low-energy tail of the ${ }^{2} \Sigma_{u}$ resonance, peaking at $4.3 \mathrm{eV}$, is not probable because of its large autodetachment width. More probable is that DEA is mediated by the high-energy tail of the ${ }^{2} \Pi_{u}$ resonance, which has the advantage of a narrow autodetachment width,

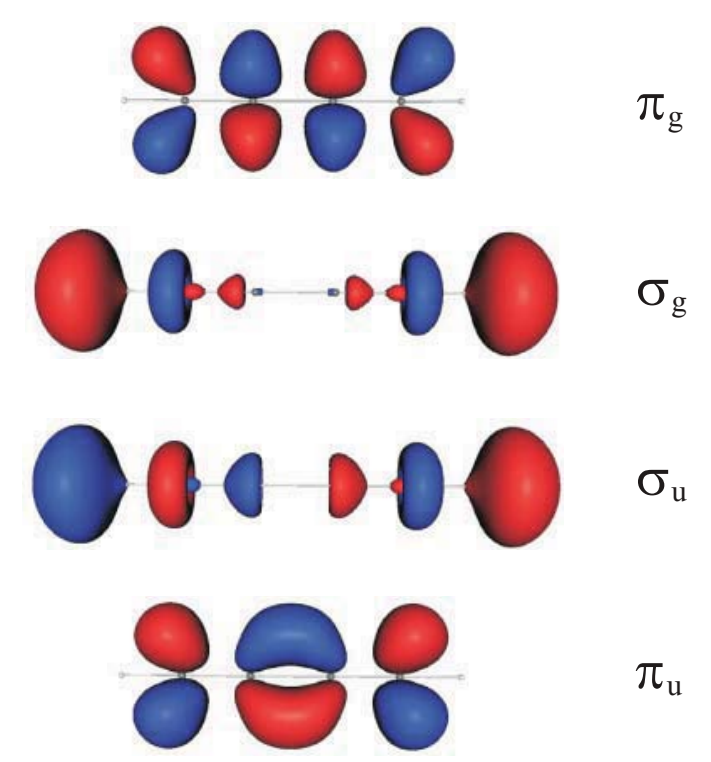

FIG. 6. (Color online) Schematic diagram of virtual SCF molecular orbitals. Drawn with the program Molekel [41]. 
and connects to the dissociative channel by $\mathrm{C}-\mathrm{H}$ bending, similar to the recently postulated case of acetylene $[42,43]$.

The situation is thus entirely analogous to that in chlorobenzene [38] where the a priori directly dissociative $\mathrm{C}-\mathrm{Cl} \sigma^{*}$ resonance, identified at $2.3 \mathrm{eV}$ by the selectivity of vibrational excitation, does not yield any $\mathrm{Cl}^{-}$signal because of its too large autodetachment width in the Franck-Condon region. DEA is mediated by the lower-lying $\pi^{*}$ resonance, having a much narrower autodetachment width, which is not directly dissociative, but connects to the dissociative channel by outof-plane bending.

The stronger, 5.25-eV peak in DEA (Fig. 5) appears to be due neither to the $6.2-\mathrm{eV}^{2} \Pi_{g}$ resonance, nor to the $6.8-\mathrm{eV} \sigma^{*}$ resonance, both because the energies do not fit, and because the autodetachment widths are too large. We notice, however, that two ${ }^{1}\left(\pi_{g}, \pi_{u}^{*}\right)$ excited states $\left({ }^{1} \Sigma\right.$ and $\left.{ }^{1} \Delta_{u}\right)$ are found in the $4.8-5.3 \mathrm{eV}$ region $[44,45]$, likely to give rise to ${ }^{2}\left(\pi_{g}, \pi_{u}^{2}\right)$ core-excited resonances, for example, ${ }^{2} \Pi_{g}$. This resonance could lie below its parent state, that is, be a valence Feshbach resonance, and have an autodetachment width narrow enough to mediate DEA. We wish to point out that the ${ }^{2}\left(\pi_{g}\right)$ shape resonance and the ${ }^{2}\left(\pi_{g}, \pi_{u}^{2}\right)$ core-excited configurations have the same symmetry, will experience configuration interaction (CI) and will mix to yield resonances of mixed shape and core-excited character. Configuration mixing in negative-ion resonances was invoked as early as 1975 by Nenner and Schulz [46]; experimental evidence was obtained by the observation of the decay of a nominally shaped 4.8-eV resonance into the lowest electronically excited state in benzene [47]. The configuration mixing was included in high-level $a b$ initio scattering calculations by Winstead and McKoy [48,49].

\section{Cross sections for vibrational excitation}

Figures 7 and 8 show the cross sections for the excitation of several vibrational states at $45^{\circ}$ and $180^{\circ}$ as a function of the incident electron energy. (The cross sections were measured also at $20^{\circ}, 90^{\circ}$, and $135^{\circ}$ but are not shown here for brevity.) The two IR-active vibrations $v_{9}\left(\mathrm{CCC}\right.$ bend) and $\nu_{8}(\mathrm{C}-\mathrm{H}$ bend) are strongly excited at threshold, as expected, at small scattering angles. The overtone of the $\mathrm{C}-\mathrm{H}$ bend vibration $2 v_{8}$ and the $\mathrm{C} \equiv \mathrm{C}$ stretch vibration $\nu_{2}$ (and, of course, its overtone $2 v_{2}$ ), being IR inactive, do not have such a threshold peak. The ${ }^{2} \Pi_{u}$ resonance appears only as shoulders on the threshold peaks. Peaks appear in the 5-7 eV region, the domain of the ${ }^{2} \Pi_{g}$ resonance, but their energy varies more than expected. This may indicate two resonances or a complex dynamics on the ${ }^{2} \Pi_{g}$ resonance surface which splits into an upper and a lower branch upon bending. The ${ }^{2} \Pi_{u}$ resonance dominates the $\mathrm{C}-\mathrm{H}$ bend and $\mathrm{C} \equiv \mathrm{C}$ stretch cross sections. It shows boomerang structures with depth varying in the different final channels. At $180^{\circ}$ the threshold peaks are missing even for the IR-active vibrations, as expected.

The boomerang structure is shown in detail in Fig. 9. The $1-\mathrm{eV}$ band and the boomerang structure are weak in the single quantum of the $\mathrm{C}-\mathrm{H}$ bend excitation, but pronounced in the double quanta, in line with the conclusion that excitation of even quanta is preferred. The spacing of the boomerang peaks is $\sim 90 \mathrm{meV}$, and is assigned to the $\mathrm{C}-\mathrm{H}$ bend motion. This

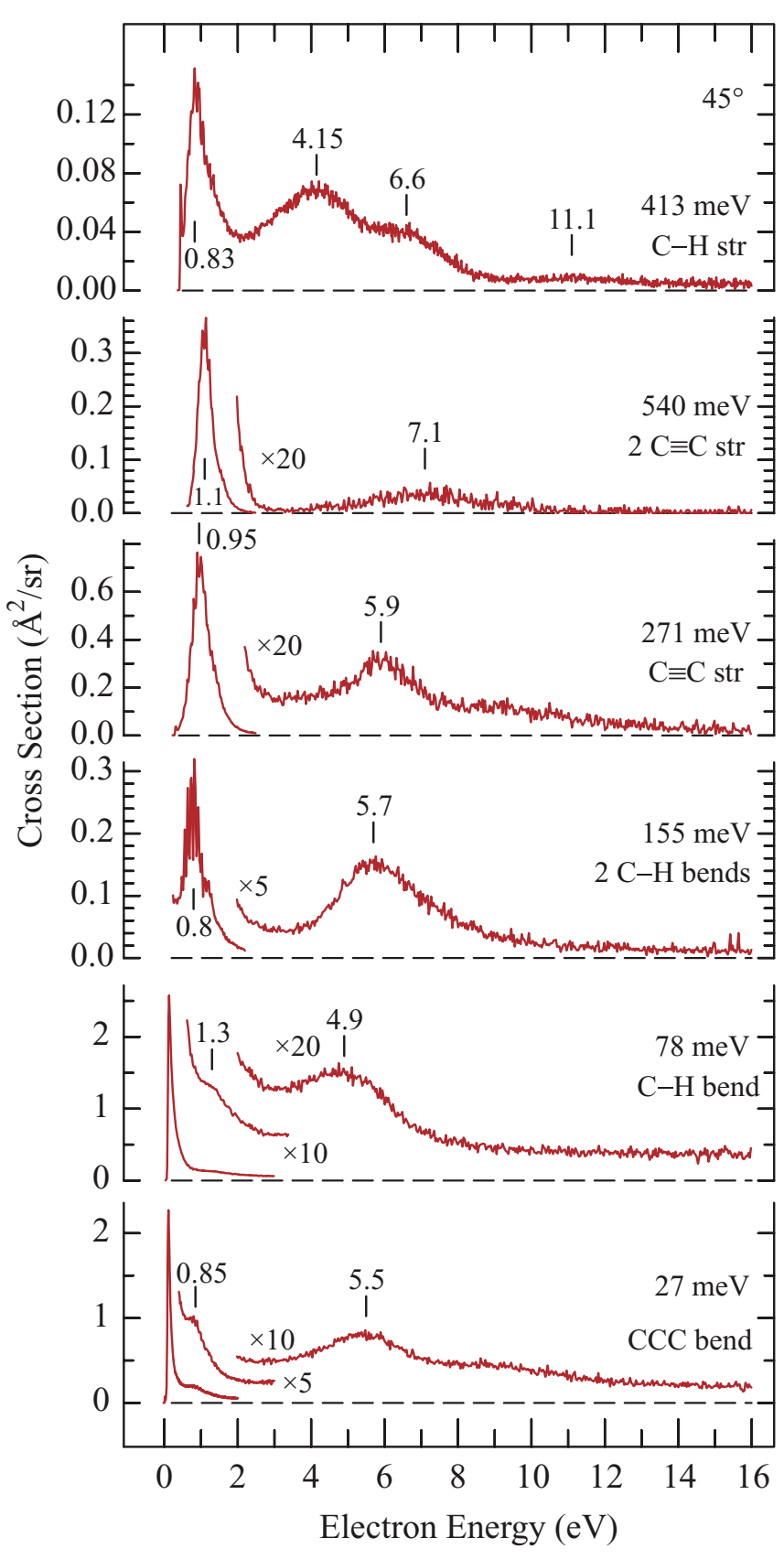

FIG. 7. (Color online) Cross sections for vibrational excitation recorded at $45^{\circ}$.

would indicate an increase of the bending vibration energy by $15 \%$ in the anion.

The IR spectrum of the nonlinear diacetylene anion trapped in solid Ar has been reported [26] with a $\mathrm{C}-\mathrm{H}$ (in-plane) bending frequency of $594.5 \mathrm{~cm}^{-1}$. For the trans $\left(C_{2 h}\right)$ anion this corresponds to the IR-active $B_{u}$ vibration. The vibration active in the boomerang motion in the present work is expected to be a different vibration, however, the IR-inactive $A_{g}$ vibration, where the anion moves from the linear to the strongly bent and back to linear geometry. The experimental frequency for this vibration is not known, but our DFT $6-31++\mathrm{G}(2 d, 2 p)$ calculation yields a value of $770 \mathrm{~cm}^{-1}$ (comparing well with $753 \mathrm{~cm}^{-1}$ from a DFT 6-311++ $\mathrm{G}^{* *}$ calculation in Ref. [26]), which is $18 \%$ higher than the value of the $\pi_{g} \mathrm{C}-\mathrm{H}$ bend 


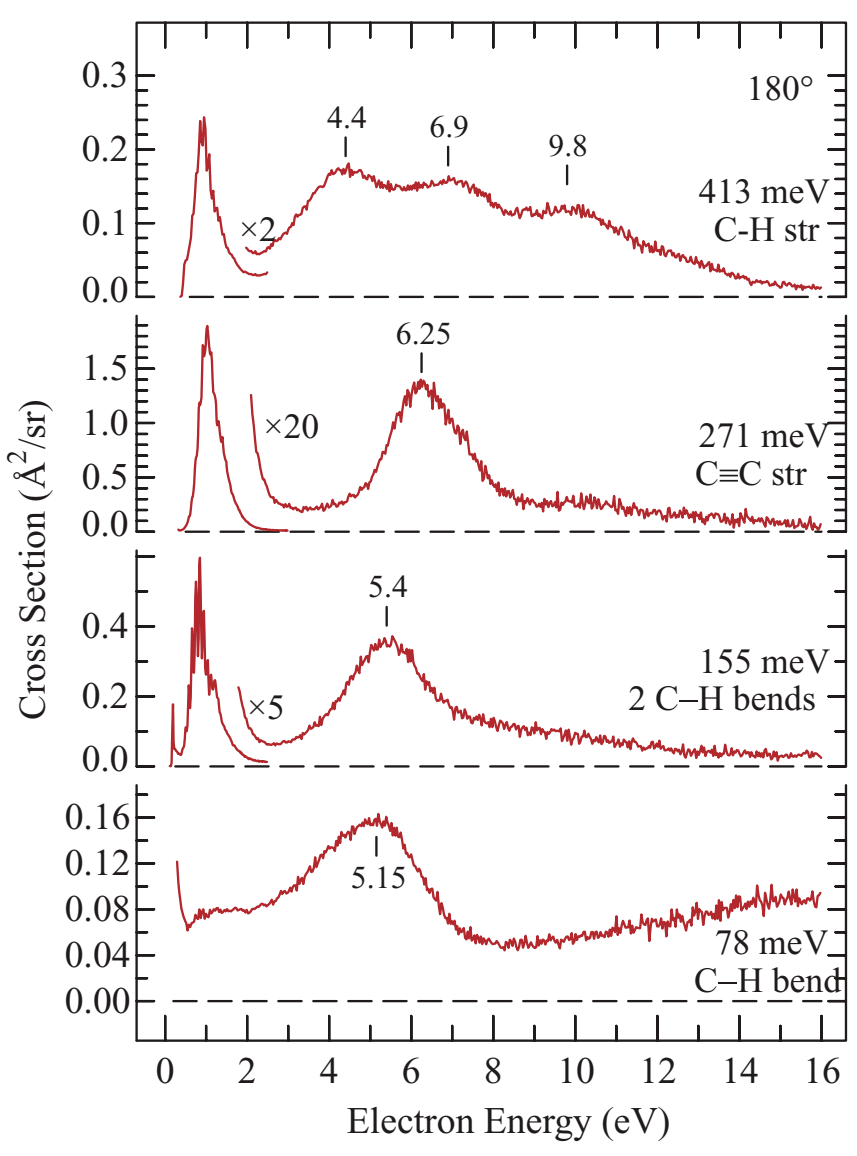

FIG. 8. (Color online) Cross sections for vibrational excitation recorded at $180^{\circ}$.

vibration for neutral acetylene (calculated with the same basis set to be $655 \mathrm{~cm}^{-1}$ ). This compares well with the observed increase of $15 \%$.

The lowest discernible boomerang structure is at $0.42 \mathrm{eV}$, in the two $\mathrm{C}-\mathrm{H}$ bend cross section in Fig. 9. This number is the adiabatic electron attachment energy. It is, strictly speaking, only the upper limit because it cannot be entirely excluded that another, very weak, boomerang structure is at even lower energies, but is too weak to be detected.

The boomerang structure in the $\mathrm{C} \equiv \mathrm{C}$ stretch and two $\mathrm{C} \equiv \mathrm{C}$ stretch excitation cross sections is less pronounced than in the two $\mathrm{C}-\mathrm{H}$ bend excitation cross section. The probable reason is that the $\mathrm{C} \equiv \mathrm{C}$ stretch vibration reveals boomerang motion along two dimensions, both the $\mathrm{C}-\mathrm{H}$ bend and the $\mathrm{C} \equiv \mathrm{C}$ stretch, leading to band congestion. The boomerang structure is more pronounced in the cross section for the excitation of $\mathrm{C} \equiv \mathrm{C}$ stretch combined with two $\mathrm{C}-\mathrm{H}$ bends. The boomerang structure is narrow and pronounced in the cross section for the excitation of $\mathrm{C}-\mathrm{C}$ stretch vibration. The spacing is also $90 \mathrm{meV}$, however, which is characteristic of $\mathrm{C}-\mathrm{H}$ bending.

\section{E. Angular distribution of vibrational excitation}

Figure 10 shows the angular distributions, recorded within the $1-\mathrm{eV}{ }^{2} \Pi_{u}$ resonance for five representative vibrational states. The excitation of the single quantum of $\mathrm{C}-\mathrm{H}$ bend is characterized by an immense $\left(21 \AA^{2} /\right.$ sr, see also Fig. 2) forward peak, due doubtlessly to direct excitation of this

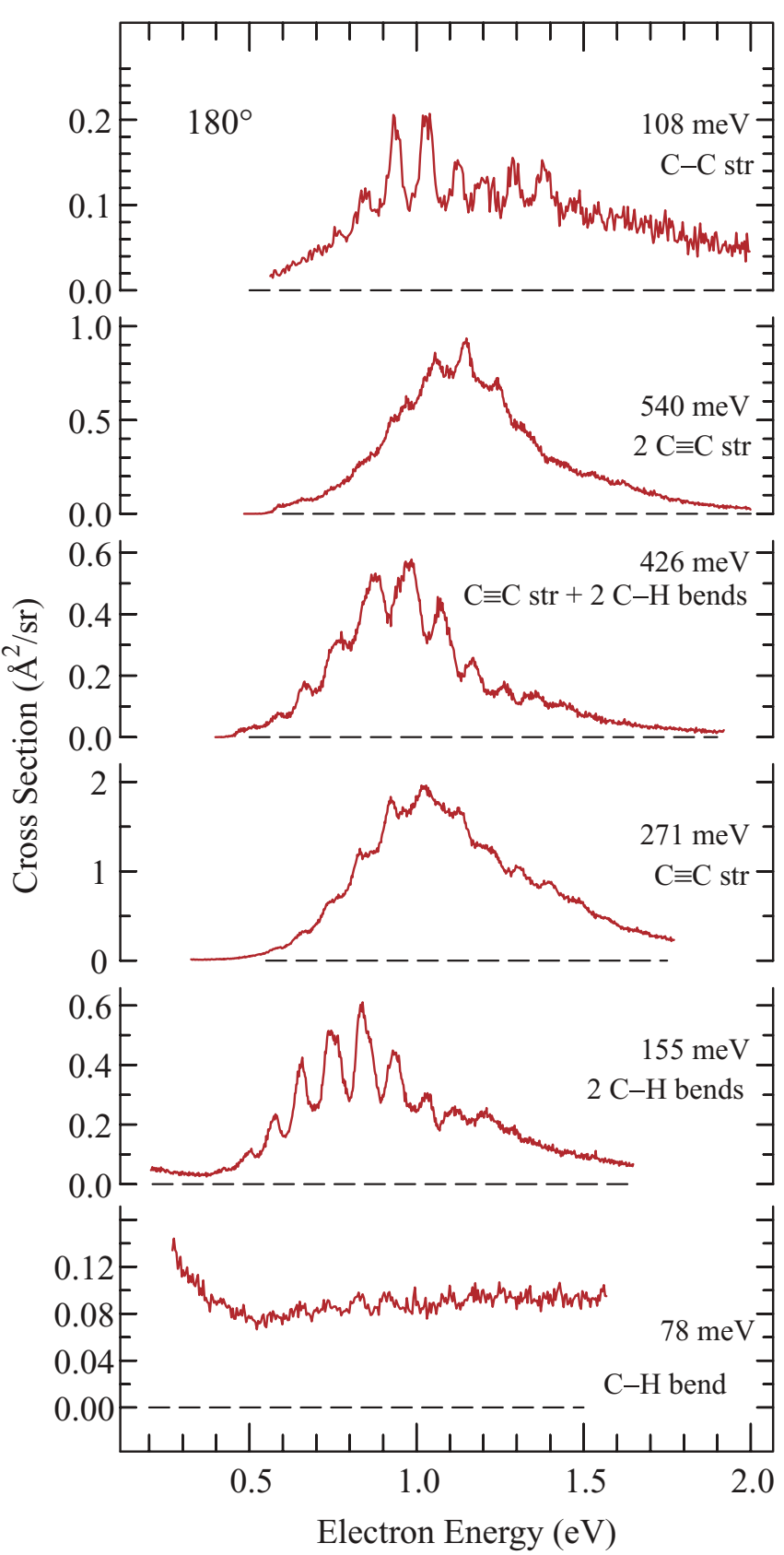

FIG. 9. (Color online) Cross sections for vibrational excitation recorded at $180^{\circ}$ on a shorter energy range,- in the ${ }^{2} \Pi_{u}$ resonance region.

IR-active vibration. A much weaker forward peak is found even for the excitation of the first overtone of this vibration, presumably because direct excitation of the overtone is also weakly IR allowed, a consequence of anharmonicity.

Apart from the forward peaks, two distinct behaviors are found: The cross sections have a minimum at $\sim 90^{\circ}$ when no or two quanta of the $\mathrm{C}-\mathrm{H}$ bend are excited, and it has a maximum at $\sim 90^{\circ}$ when a single quantum of $\mathrm{C}-\mathrm{H}$ bend is (co-)excited. The behavior is doubtlessly related to the symmetries of the resonance and of the final vibrational states. A minimum $\sim 90^{\circ}$ is obtained for excitation of $\sigma_{g}$ vibrational states, that is, the $\mathrm{C} \equiv \mathrm{C}$ stretch excitation $\nu_{2}$, even quanta of the $\pi_{g}\left(\mathrm{and} / \mathrm{or} \pi_{u}\right)$ vibrations $v_{6,8}$, or a combination of the two. A maximum $\sim 90^{\circ}$ 


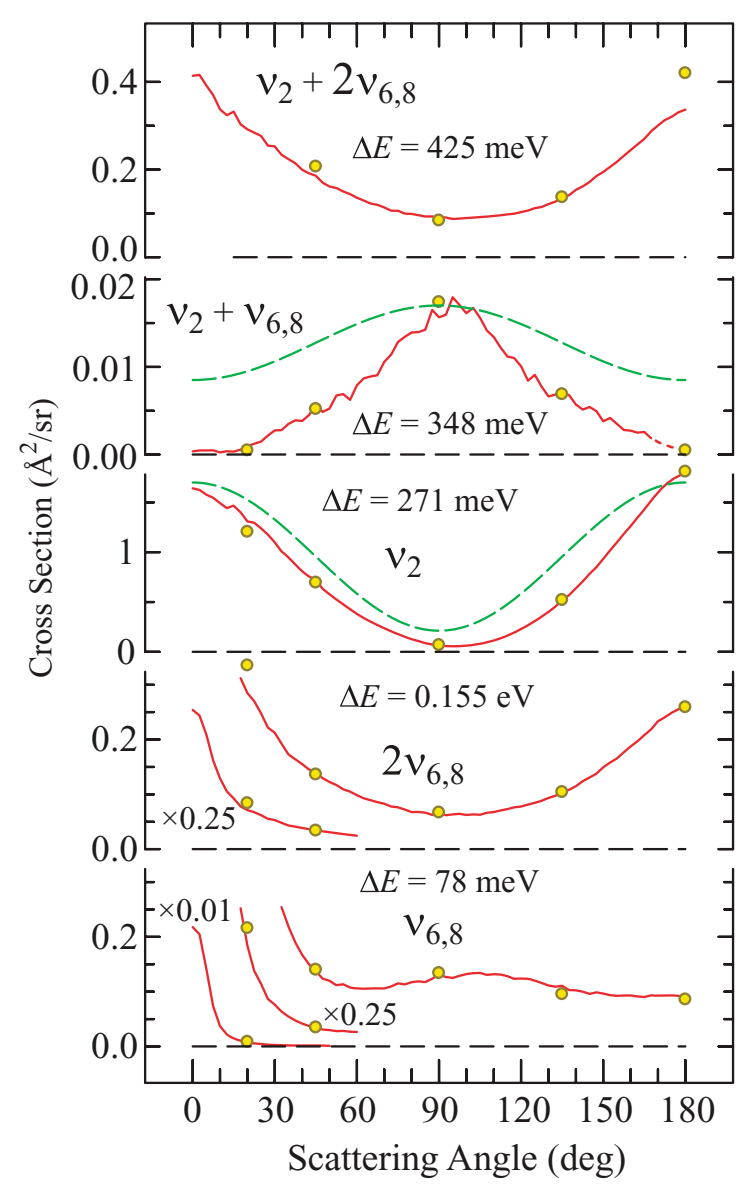

FIG. 10. (Color online) Angular distributions of the vibrational excitation cross sections at $1 \mathrm{eV}$. The (yellow) circles are the results of individual absolute measurements by the relative flow method. The (red) curves are the results of magnetic angular scans, normalized to the yellow circles. The dashed (green) lines are the predictions of the Andrick-Read theory.

is obtained for excitation of $\pi_{g}$ (and/or $\pi_{u}$ ) vibrational states, particularly well visible in combination with the $\nu_{2}$ vibration, free from the disturbing forward peak. This particular angular distribution shows a very particular feature- the cross section drops, within experimental error, to zero at $0^{\circ}$ and $180^{\circ}$. This is also well visible in the energy-loss spectra in Fig. 2. A similar observation has already been made by Trajmar and co-workers for $\mathrm{CO}_{2}$, where the symmetries of the resonance and the vibrational states are the same as in the present case (except that there is no $\pi_{g}$ vibration) [50,51].

We attempted to understand our observations using the theory of Read [52] and Andrick and Read [53]. It predicts a $\left(1+7 \cos ^{2} \theta\right)$ distribution for $\mathrm{a}^{2} \Pi_{u}$ resonance and a $\sigma_{g}$ final vibrational state, in good agreement with the angular distribution observed for the $\mathrm{C} \equiv \mathrm{C}$ stretch excitation $v_{2}$, and a qualitative agreement with that observed for $2 v_{6,8}$ excitation and for $v_{2}+2 v_{6,8}$ excitation (Fig. 10). The theory of Andrick and Read predicts $\left(2-\cos ^{2} \theta\right)$ for $\pi_{g}\left(v_{6}\right.$ and $\left.\nu_{2}+v_{6}\right)$ final states and an isotropic distribution for a $\pi_{u}\left(v_{8}\right)$ final state. The former agrees qualitatively in terms of a maximum at $90^{\circ}$, but it does not decrease to zero at $0^{\circ}$ and $180^{\circ}$.

It is interesting to note that entirely different angular distributions were observed in the closely related case of acetylene
TABLE II. Integral VE cross sections at $E=1 \mathrm{eV}$.

\begin{tabular}{lccccc}
\hline \hline State & $v_{6,8}$ & $2 v_{6,8}$ & $v_{2}$ & $v_{2}+v_{6,8}$ & $v_{2}+2 v_{6,8}$ \\
\hline ICS $\left(\AA^{2}\right)$ & 3.05 & 1.45 & 5.81 & 0.12 & 1.81 \\
\hline \hline
\end{tabular}

[54], although the vibrations have the same symmetries and the only difference in symmetry is that the shape resonance is ${ }^{2} \Pi_{g}$ and not ${ }^{2} \Pi_{u}$. In acetylene the angular distributions were the same for all final vibrational states and could be reproduced by Andrick and Read theory, but only when the reduced symmetry of the bent resonance was considered. This strategy was not successful, however, in the present case of diacetylene.

With respect to the vanishing cross sections at $0^{\circ}$ and $180^{\circ}$, Cartwright and Trajmar [51] proposed, for the similar case of $\mathrm{CO}_{2}$, that $\pi$ vibrations, containing odd quanta of bending, are excited by a mechanism involving core-excited resonances with a $\Sigma^{-}$symmetry, so that the symmetry selection rule forbidding $\Sigma^{+} \leftrightarrow \Sigma^{-}$transitions at $0^{\circ}$ and $180^{\circ} \quad[55,56]$ applies. We note that in our case there is a potential ${ }^{1} \Sigma_{u}^{-}$parent state for such a resonance $[44,45]$ (the extra electron could be captured in a diffuse $3 s$-like $\sigma_{g}$ orbital in the resonance), but that it is at $4.8 \mathrm{eV}$, too high for a vertical electron capture at $1 \mathrm{eV}$. A $1-\mathrm{eV}$ electron must be, at least initially, captured into the ${ }^{2} \Pi_{u}$ shape resonance. The core-excited resonance could enter into play at strongly bent geometries, if its energy would descend faster than that of the ${ }^{2} \Pi_{u}$ shape resonance, with which it could then mix.

Integral cross sections were obtained by integration under the angular distributions and are given in Table II. Note that the $v_{2}+2 v_{6,8}$ cross section is much larger than the $v_{2}+v_{6,8}$ cross section. (In fact, the latter is smaller than the former even at $90^{\circ}$, where it peaks.) The conclusion of the early study [14] reported the strong propensity for exciting double quanta of the bending vibration is thus confirmed by the present angle-resolved study.

\section{CONCLUSIONS}

The selectivity of vibrational excitation permitted unique insight into the resonant structure of diacetylene. In particular, the selective excitation of the $\mathrm{C}-\mathrm{H}$ stretch vibration revealed three $\sigma^{*}$ shape resonances at $4.3,6.8$, and $9.8 \mathrm{eV}$. The 4.3-eV band is suggested to be the ${ }^{2} \Sigma_{u}$ resonance, with a temporary electron capture in the LUMO-1 $\sigma_{u}$ orbital. In addition, there is the ${ }^{2} \Pi_{u}$ shape resonance at $1 \mathrm{eV}$, which excites strongly the $\mathrm{C} \equiv \mathrm{C}$ stretch and the $\mathrm{C}-\mathrm{H}$ bend vibrations, and the ${ }^{2} \Pi_{g}$ shape resonance at $6.2 \mathrm{eV}$, both of which were already reported earlier [14]. The shapes of the observed bands indicate autodetachment widths of $\sim 30 \mathrm{meV}$ for the $1-\mathrm{eV}^{2} \Pi_{u}$ resonance, and $\sim 2 \mathrm{eV}$ for the remaining shape resonances.

This information permits a deeper discussion of the mechanism of DEA, the absolute cross section of which we reported recently [10]. Although both the high-energy tail of the ${ }^{2} \Pi_{u}$ resonance and the low-energy tail of the $4.3-\mathrm{eV}^{2} \Sigma_{u}$ resonance are possible candidates for the weak $\left(3 \mathrm{pm}^{2}\right) 2.5-\mathrm{eV}$ DEA band, the large observed width of the $\sigma^{*}$ resonance leads to the conclusion that the ${ }^{2} \Pi_{u}$ resonance, with its narrow width, acts as a doorway state and couples to the dissociative channel via $\mathrm{C}-\mathrm{H}$ bending. The prominence of the $\mathrm{C}-\mathrm{H}$ bending excitation in the ${ }^{2} \Pi_{u}$ resonance region indicates that $\mathrm{C}-\mathrm{H}$ bending is the 
primary relaxation path and supports this interpretation. The strong excitation of the $\mathrm{C}-\mathrm{H}$ bending is an evidence for the $c$ is and trans bent equilibrium structures of the radical anion. This is the same DEA mechanism as that recently postulated theoretically for acetylene [42,43], and in line with our earlier conclusions [10].

In this respect it is interesting to note that these findings are related to a current discussion on DEA mechanisms in the literature. The mechanism proposed here for diacetylene appears to be quite frequent, and is found, for example, in the prototype case of chlorobenzene, where the selectivity of vibrational excitation, as in the present case, permitted unambiguous identification of the $\pi^{*}$ and $\sigma^{*}$ resonances, and where the energy of the DEA band clearly indicates the $\pi^{*}$ and not the $\sigma^{*}$ resonance as the doorway state [38].

Direct dissociation in the $\sigma$ symmetry is possible, however, and is doubtlessly the mechanism in saturated compounds which have no $\pi^{*}$ resonances. It may yield sizable cross sections, as, for example, in $\mathrm{HBr}$ or $\mathrm{HCl}$ [57], where both VE and DEA are well understood theoretically [58,59]. On the other hand, the mechanism of low-energy DEA to unsaturated compounds which have a sizable dipole moment, where both a $\pi^{*}$ resonance and a dipole-bound resonance may be present and could act as a doorway state, is still being discussed [60-64].

None of the resonances observed in the present VE study explains the more intense $\left(73 \mathrm{pm}^{2}\right)$ DEA band at $5.25 \mathrm{eV}$, both in terms of energy and in terms of autodetachment width, and we conclude that this DEA band must be due to core-excited resonance(s), with one of the $\left(\pi, \pi^{*}\right)$ valence excited states $\sim 5 \mathrm{eV}[44,45]$ as the parent state. We point out, however, that at least some resonances in the 5-eV range are likely to be affected by configuration mixing, since the ${ }^{2}\left(\pi_{g}\right)$ shape and the ${ }^{2}\left(\pi_{g}, \pi_{u}^{2}\right)$ core-excited configurations have the same symmetry (and multiplicity) and can mix.

The resonant excitation of the $\mathrm{C}-\mathrm{H}$ bend vibrations has a strong preference for excitation of double quanta, understandable in terms of symmetries of the final vibrational states.
This propensity is best visible when bending is superimposed on one or several quanta of the $\mathrm{C} \equiv \mathrm{C}$ stretch, and is thus not affected by direct excitation of IR-active vibrations. As an example, the integral cross section for the excitation of $v_{2}+2 v_{6,8}$ is $1.81 \AA^{2}, 15 \times$ larger than the cross section for $v_{2}+v_{6,8}$ excitation (Table II). This propensity is not limited to diacetylene; it was also found for the excitation of the $\pi_{u}$ bending vibration through the ${ }^{2} \Pi$ resonance in OCS [30]. An interesting question is whether this propensity affects the DEA cross section, which could be the case, because the VE and the DEA channels compete and interchannel coupling is possible.

The symmetry of the final vibrational state is strongly reflected not only in the magnitude, but also in the angular distributions of the odd and even quanta of the $\mathrm{C}-\mathrm{H}$ bending vibration via the ${ }^{2} \Pi_{u}$ resonance at $1 \mathrm{eV}$. The angular distributions of the $\sigma_{g}$ vibrations, either $\nu_{2} \mathrm{C} \equiv \mathrm{C}$ stretch, or even quanta of $v_{6,8} \mathrm{C}-\mathrm{H}$ bend, or a combination of the two, have a minimum at $90^{\circ}$, and are well reproduced by the the Andrick-Read theory [52,53]. The angular distributions of the $\pi$ vibrations, odd quanta of $\mathrm{C}-\mathrm{H}$ bend $v_{6,8}$, alone or in combination with $\nu_{2}$, are reproduced only qualitatively by the Andrick-Read theory, in the sense of having a maximum at $90^{\circ}$. The excitation of the $v_{2}+v_{6,8}$ has a peculiar property, however, similar to that reported earlier for $\mathrm{CO}_{2}[50,51]$ - the cross section drops to zero at $0^{\circ}$ and $180^{\circ}$. This property is not reproduced by the Andrick-Read theory and we feel that it is not properly understood at present, at least not for the diacetylene case.

\section{ACKNOWLEDGMENTS}

We thank Professor John P. Maier and Jacques Lecoultre for a generous sample of diacetylene. This research is part of project No. 200020-131962/1 of the Swiss National Science Foundation, of project SBF No. C07.0018 of the State Secretariat for Education and Research, and of COST Action CM0601.
[1] J. Cernicharo, M. Guelin, M. Agundez, K. Kawaguchi, M. McCarthy, and P. Thaddeus, Astron. Astrophys. 467, L37 (2007)

[2] H. Gupta, S. Brünken, F. Tamassia, C. A. Gottlieb, M. C. McCarthy, and P. Thaddeus, Astrophys. J. 655, L57 (2007).

[3] D. E. Shemansky, A. I. F. Stewart, R. A. West, L. W. Esposito, J. T. Hallett, and X. Liu, Science 308, 978 (2005).

[4] M. Burgdorf, G. Orton, J. van Cleve, V. Meadows, and J. Houck, Icarus 184, 634 (2006).

[5] J. Benedikt, A. Consoli, M. Schulze, and A. von Keudell, J. Phys. Chem. A 111, 10453 (2007).

[6] M. Mao, J. Benedikt, A. Consoli, and A. Bogaerts, J. Phys. D 41, 225201 (2008).

[7] X. Gu, Y. Guo, A. M. Mebel, and R. I. Kaiser, J. Phys. Chem. A 110, 11265 (2006).

[8] X. Gu, Y. Guo, A. M. Mebel, and R. I. Kaiser, Combust. Flame 151, 245 (2007).
[9] A. D'Anna, M. Alfe, B. Apicella, A. Tregrossi, and A. Ciajolo, Energy Fuels 21, 2655 (2007).

[10] O. May, J. Fedor, B. C. Ibănescu, and M. Allan, Phys. Rev. A 77, 040701(R) (2008).

[11] M. Allan, E. Kloster-Jensen, and J. P. Maier, Chem. Phys. 7, 11 (1976).

[12] H. Estrada, L. S. Cederbaum, and W. Domcke, J. Chem. Phys. 84, 152 (1986).

[13] I. C. Walker, A. Stamatovic, and S. F. Wong, J. Chem. Phys. 69 , 5532 (1978).

[14] M. Allan, Chem. Phys. 86, 303 (1984).

[15] M. Allan, J. Phys. B 25, 1559 (1992).

[16] M. Allan, J. Phys. B 38, 3655 (2005).

[17] M. Allan, J. Phys. B 40, 3531 (2007).

[18] M. Allan, Phys. Rev. A 81, 042706 (2010).

[19] A. Gopalan, J. Bömmels, S. Götte, A. Landwehr, K. Franz, M. W. Ruf, H. Hotop, and K. Bartschat, Eur. Phys. J. D 22, 17 (2003). 
[20] J. C. Nickel, P. W. Zetner, G. Shen, and S. Trajmar, J. Phys. E 22, 730 (1989).

[21] R. K. Nesbet, Phys. Rev. A 20, 58 (1979).

[22] F. H. Read and J. M. Channing, Rev. Sci. Instrum. 67, 2373 (1996).

[23] M. Zubek, N. Gulley, G. C. King, and F. H. Read, J. Phys. B 29, L239 (1996).

[24] A. A. Granovsky, Firefly version 7.1.G, [http://classic. chem.msu.su/gran/firefly/index.ht ml].

[25] M. W. Schmidt, K. K. Baldridge, J. A. Boatz, S. T. Elbert, M. S. Gordon, J. H. Jensen, S. Koseki, N. Matsunaga, K. A. Nguyen, S. Su et al., J. Comput. Chem. 14, 1347 (1993).

[26] J. Szczepanski, H. Wang, B. Jones, C. A. Arrington, and M. T. Vala, PhysChemChemPhys 7, 738 (2005).

[27] T. Shimanouchi, Tables of Molecular Vibrational Frequencies Consolidated Volume I (National Bureau of Standards, Washington D.C., 1972).

[28] I. Freund and R. S. Halford, J. Chem. Phys. 42, 4131 (1965).

[29] M. Allan, J. Electron Spectrosc. Relat. Phenom. 48, 219 (1989).

[30] T. H. Hoffmann, H. Hotop, and M. Allan, J. Phys. B 41, 195202 (2008).

[31] S. F. Wong and G. J. Schulz, Phys. Rev. Lett. 35, 1429 (1975).

[32] G. A. Gallup, Phys. Rev. A 34, 2746 (1986).

[33] G. A. Gallup, J. Chem. Phys. 99, 827 (1993).

[34] G. J. Schulz, Rev. Mod. Phys. 45, 423 (1973).

[35] S. W. Staley and J. T. Strnad, J. Phys. Chem. 98, 116 (1994).

[36] D. Chen and G. A. Gallup, J. Chem. Phys. 93, 8893 (1990).

[37] A. Modelli and D. Jones, PhysChemChemPhys 13, 276 (2011).

[38] T. Skalický, C. Chollet, N. Pasquier, and M. Allan, PhysChemChemPhys 4, 3583 (2002).

[39] M. Allan and L. Andrić, J. Chem. Phys. 105, 3559 (1996).

[40] O. Schafer, M. Allan, G. Szeimies, and M. Sanktjohanser, J. Am. Chem. Soc. 114, 8180 (1992).
[41] U. Varetto, Molekel 5.4, Swiss National Supercomputing Centre, Manno, Switzerland.

[42] S. T. Chourou and A. E. Orel, Phys. Rev. A 77, 042709 (2008).

[43] S. T. Chourou and A. E. Orel, Phys. Rev. A 80, 034701 (2009).

[44] H. J. Haink and M. Jungen, Chem. Phys. Lett. 61, 319 (1979).

[45] M. Allan, J. Chem. Phys. 80, 6020 (1984).

[46] I. Nenner and G. J. Schulz, J. Chem. Phys. 62, 1747 (1975).

[47] M. Allan, Helv. Chim. Acta 65, 2008 (1982).

[48] C. Winstead and V. McKoy, Phys. Rev. Lett. 98, 113201 (2007).

[49] C. Winstead and V. McKoy, Phys. Rev. A 76, 012712 (2007).

[50] D. F. Register, H. Nishimura, and S. Trajmar, J. Phys. B 13, 1651 (1980).

[51] D. C. Cartwright and S. Trajmar, J. Phys. B 29, 1549 (1996).

[52] F. H. Read, J. Phys. B: At. Mol. Phys. 1, 893 (1968).

[53] D. Andrick and F. H. Read, J. Phys. B 4, 389 (1971).

[54] L. Andrić and R. I. Hall, J. Phys. B 21, 355 (1988).

[55] D. C. Cartwright, S. Trajmar, W. Williams, and D. L. Huestis, Phys. Rev. Lett. 27, 704 (1971).

[56] W. A. Goddard III, D. L. Huestis, D. C. Cartwright, and S. Trajmar, Chem. Phys. Lett. 11, 329 (1971).

[57] J. Fedor, O. May, and M. Allan, Phys. Rev. A 78, 032701 (2008).

[58] M. Č́̌žek, J. Horáček, A.-Ch. Sergenton, D. B. Popović, M. Allan, W. Domcke, T. Leininger, and F. X. Gadea, Phys. Rev. A 63, 062710 (2001).

[59] J. Fedor, C. Winstead, V. McKoy, M. Čížek, K. Houfek, P. Kolorenč, and J. Horáček, Phys. Rev. A 81, 042702 (2010).

[60] T. N. Rescigno, C. S. Trevisan, and A. E. Orel, Phys. Rev. Lett. 96, 213201 (2006).

[61] G. A. Gallup, P. D. Burrow, and I. I. Fabrikant, Phys. Rev. A 79, 042701 (2009).

[62] T. N. Rescigno, C. S. Trevisan, and A. E. Orel, Phys. Rev. A 80, 046701 (2009).

[63] G. A. Gallup, P. D. Burrow, and I. I. Fabrikant, Phys. Rev. A 80, 046702 (2009).

[64] I. I. Fabrikant, J. Phys. Conf. Ser. 204, 012004 (2010). 